\title{
Palm rejection algorithms on touch screen communication system
}

\author{
Giao N Pham ${ }^{1, *}$, Binh A Nguyen ${ }^{2}$, Tung V Nguyen ${ }^{2}$, Manh Hoang ${ }^{2}$ and Anh N Bui ${ }^{1}$ \\ ${ }^{1}$ Department of Computing Fundamentals, FPT University, Hanoi, Vietnam. \\ ${ }^{2}$ ICT Department, FPT University, Hanoi, Vietnam.
}

World Journal of Advanced Engineering Technology and Sciences, 2021, 02(02), 052-057

Publication history: Received on 27 March 2021; revised on 02 May 2021; accepted on 05 May 2021

Article DOI: https://doi.org/10.30574/wjaets.2021.2.2.0036

\begin{abstract}
This paper will discuss one of the most important problems in touch screen systems named palm rejection. Various solutions for palm rejection are discussed through the paper, which are based on hardware modules, software modules, and hybrid software-hardware modules. We hope that our proposed technical research with various solutions will be a great reference for engineers and scientists working on touch screen system design.
\end{abstract}

Keywords: Touchscreen panel; Touch screen controller; sensor design; ASIC analogue design; ASIC digital design; palm rejection algorithm

\section{Introduction}

Human-machine interactions are the research on the interfaces between people (users) and electronic devices, which help electronic devices to be more and more comfortable for human living [1]. The display screen of electronics device is not only the output for users but also people can use it for input commands such as a touchscreen in ATM, Laptop, game consoles, wearable devices. Hence, touchscreen system enables users to interact directly without using traditional devices such as mouse, keyboards. In addition, touchscreen systems have been improved to communicate with active pens which promises many advantage applications and give people more choices of interactions.

The popularity of smartphones, tablets, wearable devices have been driving touchscreen systems in electronics devices [2]. However, one of a main problem touchscreen system needed to resolve is palm rejection. Palm rejection is not really about your palm, it is about the subparts of palm or other object which rest on a touch screen without activating it. In this survey, we will analyze some palm rejection algorithms based on hardware, software parts of a touchscreen system.

The organization of our paper is as follows. In Section 2, we briefly introduce the preliminary of touchscreen system. In Section 3, 4, 5, and 6, the palm rejections algorithm based on resistance sensing sensors [3], multi-frequencies driving [4], driving-sensing swapping method, and touch capacitance profiles filter are discussed [5], respectively. Finally, conclusion and open discussion are given in Section 7.

\section{Preliminary}

\subsection{Overview of a touchscreen system}

The overall concept of a touchscreen system is given in figure 1 which includes three main parts: sensor system, analogue front end, and digital processing part. The sensor system is designed for both sensing and driving the signal from not only environments but also the analogue-front-end (AFE) part. AFE will handle the analogue signal filters,

* Corresponding author: Giao N Pham; Phone: +84 (0)967711286; E-mail: giaopn@fe.edu.vn

Department of Computing Fundamentals, FPT University, Hanoi, Vietnam.

Copyright (C) 2021 Author(s) retain the copyright of this article. This article is published under the terms of the Creative Commons Attribution Liscense 4.0. 
multiplexer control, analogue-to-digital (ADC) and digital-to-analogues (DAC) converters. The digital signals will first pass to some digital filters, fast Fourier transform (FFT), memory storage, then processing algorithms will be handled by MCU

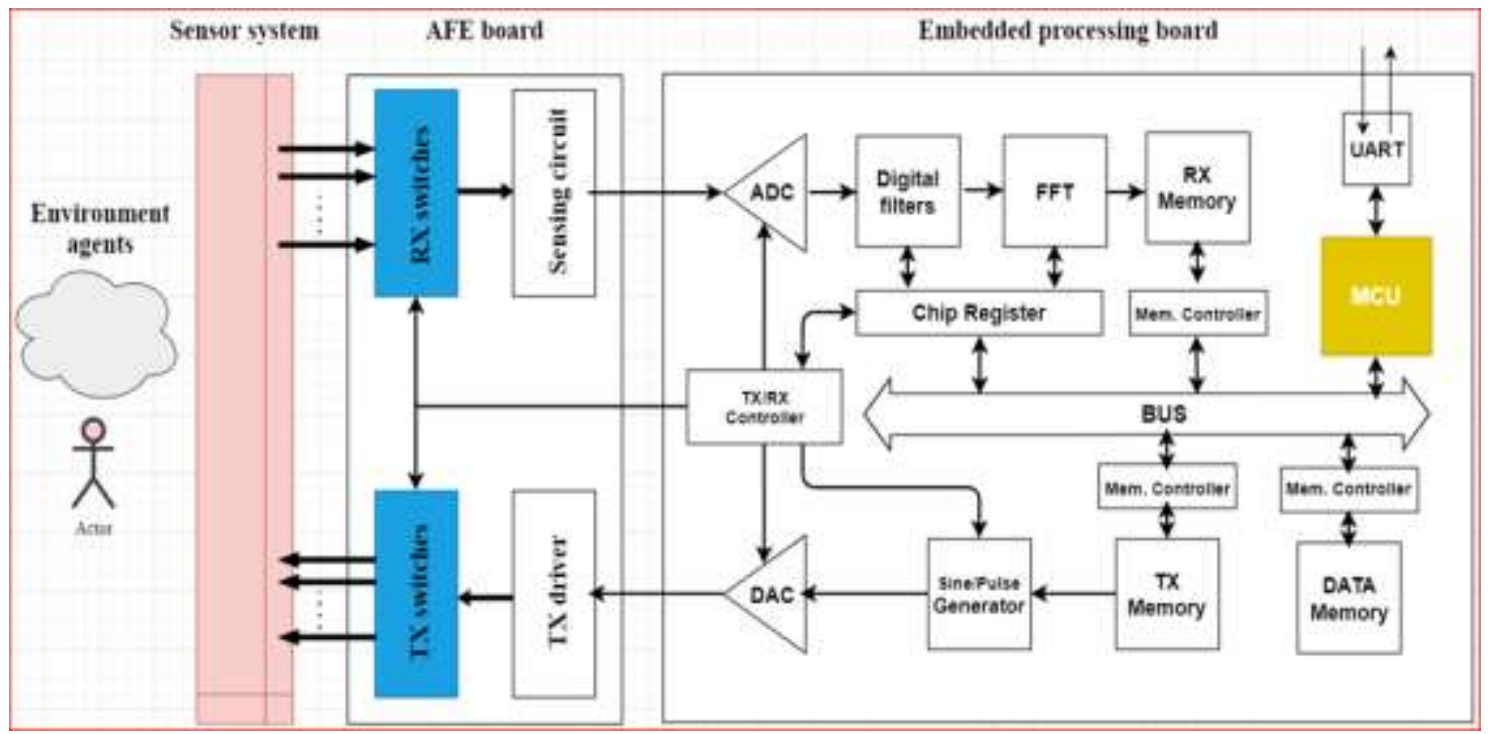

Figure 1 A full considered touch screen system with functional modules.

\subsection{Palm rejection algorithm in touch screen system}

The concept and definition of palm rejection algorithm is clearly shown in figure 2. As mentioned therein, the touchscreen controller with palm rejection algorithms will remove the capacitance profile of palm in touchscreen database. Consequently, the expected data with active pen (stylus) and fingers profiles will be available with no noise. Palm rejection algorithm is significant especially for the large screen devices since in the smaller screen, the user's hand rests mostly outside the screen. In literature, there are numerous palm rejection algorithms, during our paper, we will consider the palm rejection algorithm in sensor system, AFE board, and MCU as the highlight in figure 1

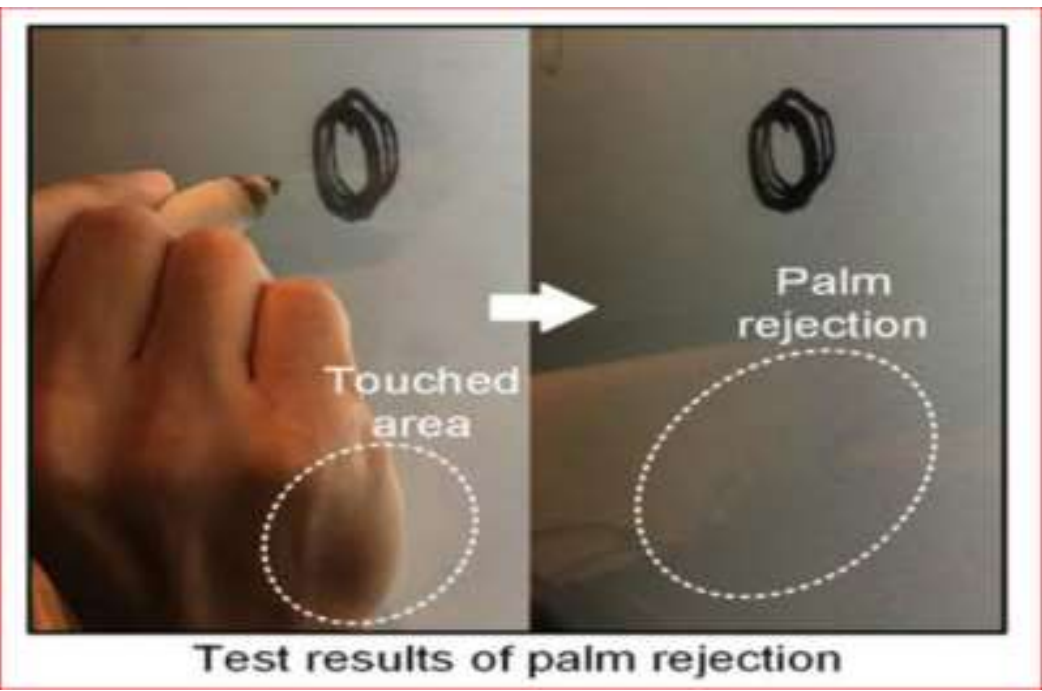

Figure 2 Palm rejection algorithm in touchscreen system

\section{Resistive touch screen}

The palm rejection algorithm in this section will be implemented in sensor system part where the resistive screen with two layers of transparent conductor ITO (indium tin oxide) is considered. Inside the screen, two layers are kept apart by some transparent space dots. Therefore, when active pens or fingers touch on the top of surface, transparent conductors make the connection and touch screen controller will based on the profile of connection for location 
determination. In the case of palm touches the surface, the numbers of connection will be bigger than in the case of pen or finger. Then, as a suitable chosen specification diameter, touch screen controller will distinguish between palm versus pen or fingers.

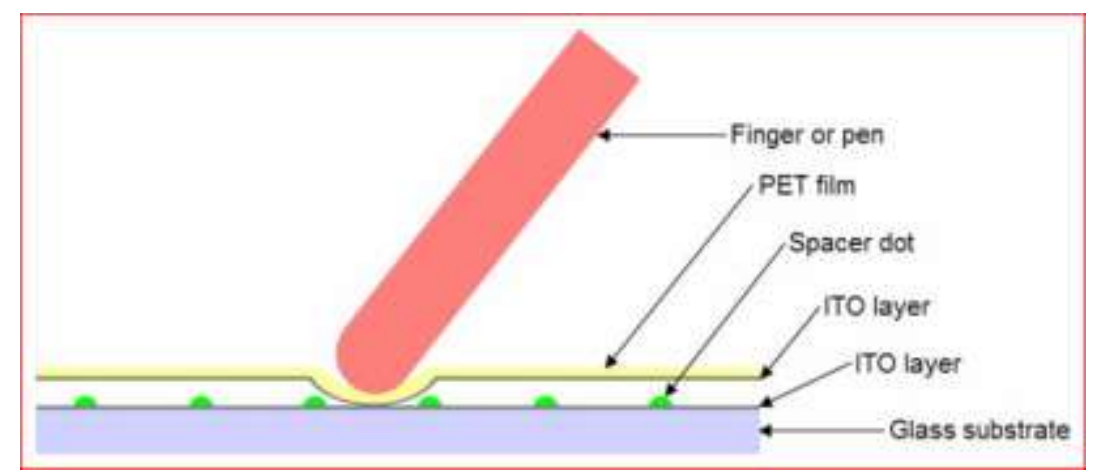

Figure 3 Resistive touch screen

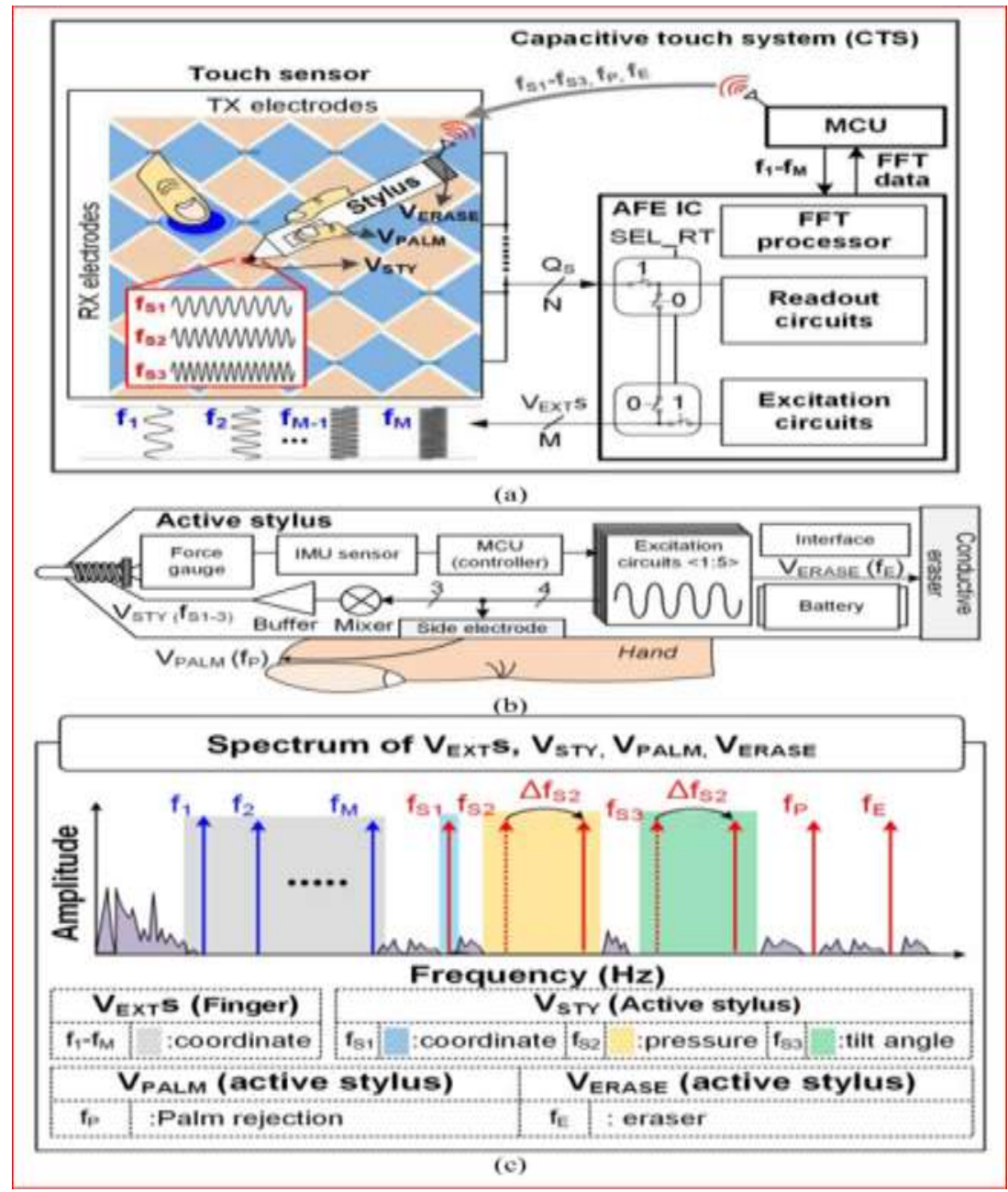

Figure 4 Capacitive touch system (a) and multi-frequencies driving method (b, c).

\section{Multi-frequencies driving algorithm}

In this section, we will consider the capacitive touchscreen where the electrodes will be classified into sensing and driving electrodes. In addition, the proposed system will use multi-frequencies for each driving electrodes. Then, the Fast Fourier Transform (FFT) module will be used in controller for frequencies analysis. Therefore, the frequency resources will be assigned properly for exciting frequencies, stylus frequencies, finger frequencies... then, the unwanted 
palm frequencies will be determined, and palm rejection algorithm will be implemented in MCU via FFT data where frequency filters is used to remove the unwanted frequencies for further processing as clearly explained in figure 4.

\section{Swapping algorithm}

In this section, we will propose the method to distinguish stylus from palm named swapping of sensing and driving electrodes. The core idea of swapping method is from the capacitance profile of palm is much larger than profile area of stylus; hence for the worst case, the sensing profile of stylus or palm will be hidden inside the profile area of palm. To avoid the burring of expected profile, the swapping method between sensing and driving electrodes as figure 5 are a simple but a good solution. The simulation of swapping method has been shown in figure 6 where swapping of A-mode B-mode to resolve the problem of hidden profile area. The swapping algorithm is handled by AFE part.

\section{Filter algorithm}

In this section, we will propose the method to distinguish finger from palm named filter on sensing areas. The core idea of filter method is from the capacitance profile of palm is much larger than profile area of fingers. The flow diagram of filter algorithm is shown steps by steps in figure 7. The demonstration with validation sensing profile of finger and palm is shown in figure 8. The filter algorithm is handled by MCU.

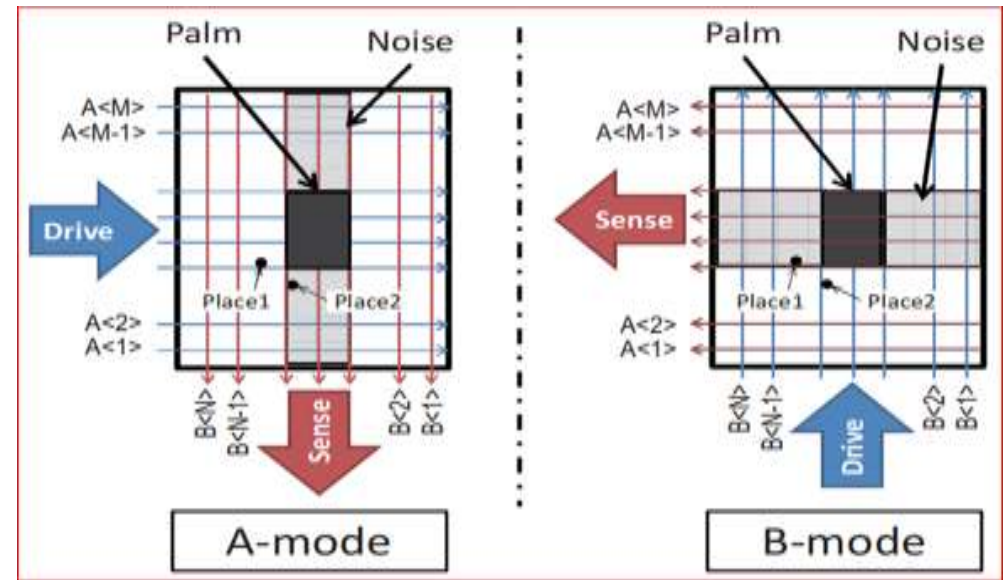

Figure 5 Swapping between driving and sensing

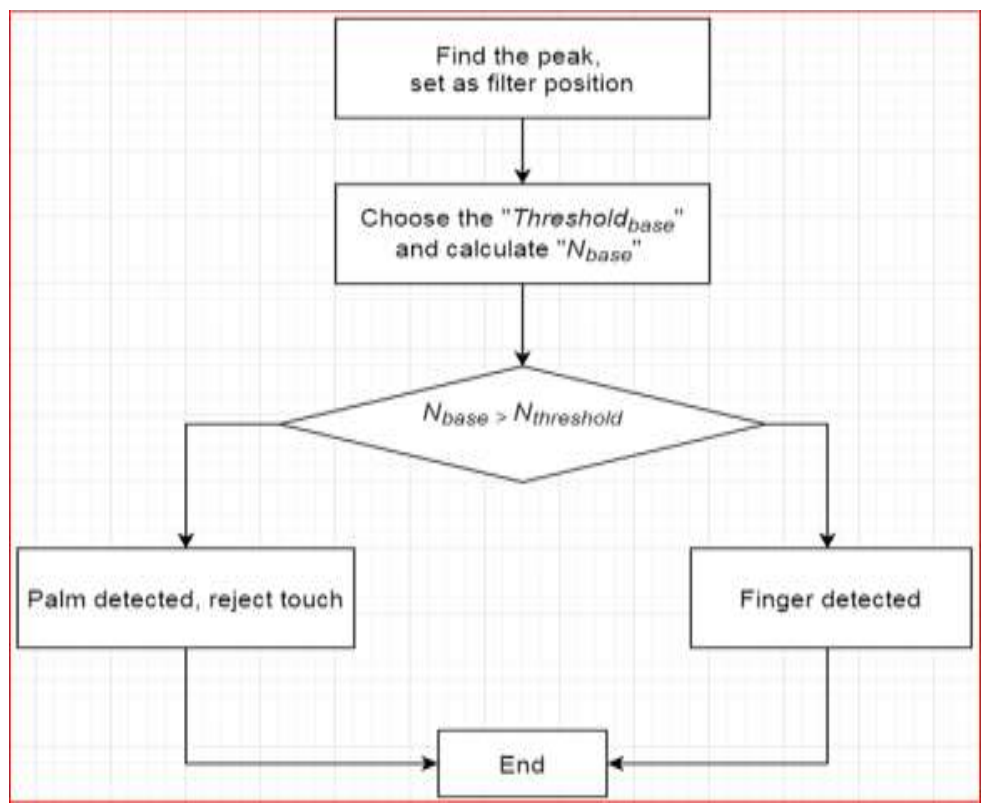

Figure 6 Filter method flow diagram 


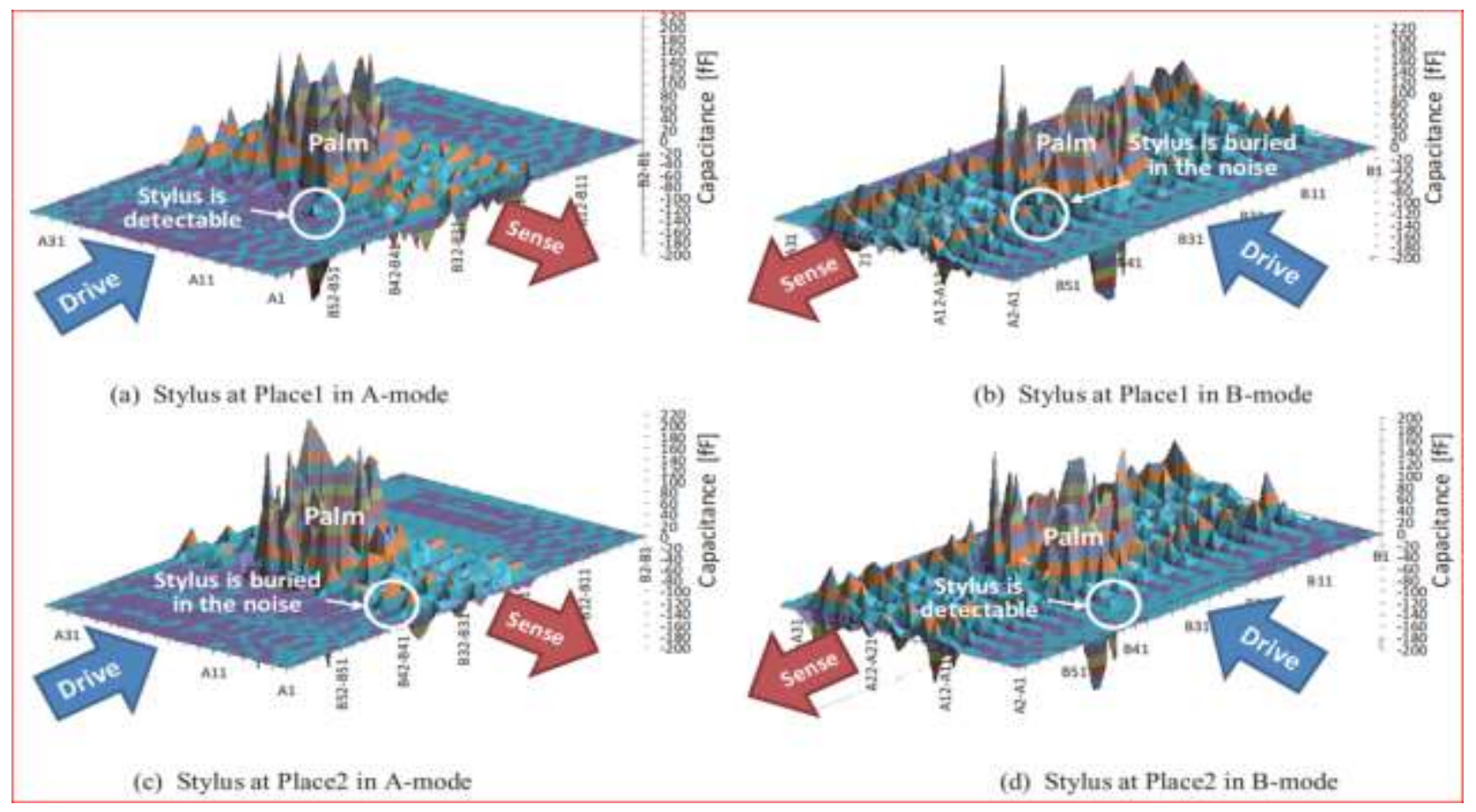

Figure 7 Operation of swapping sensing and driving method.

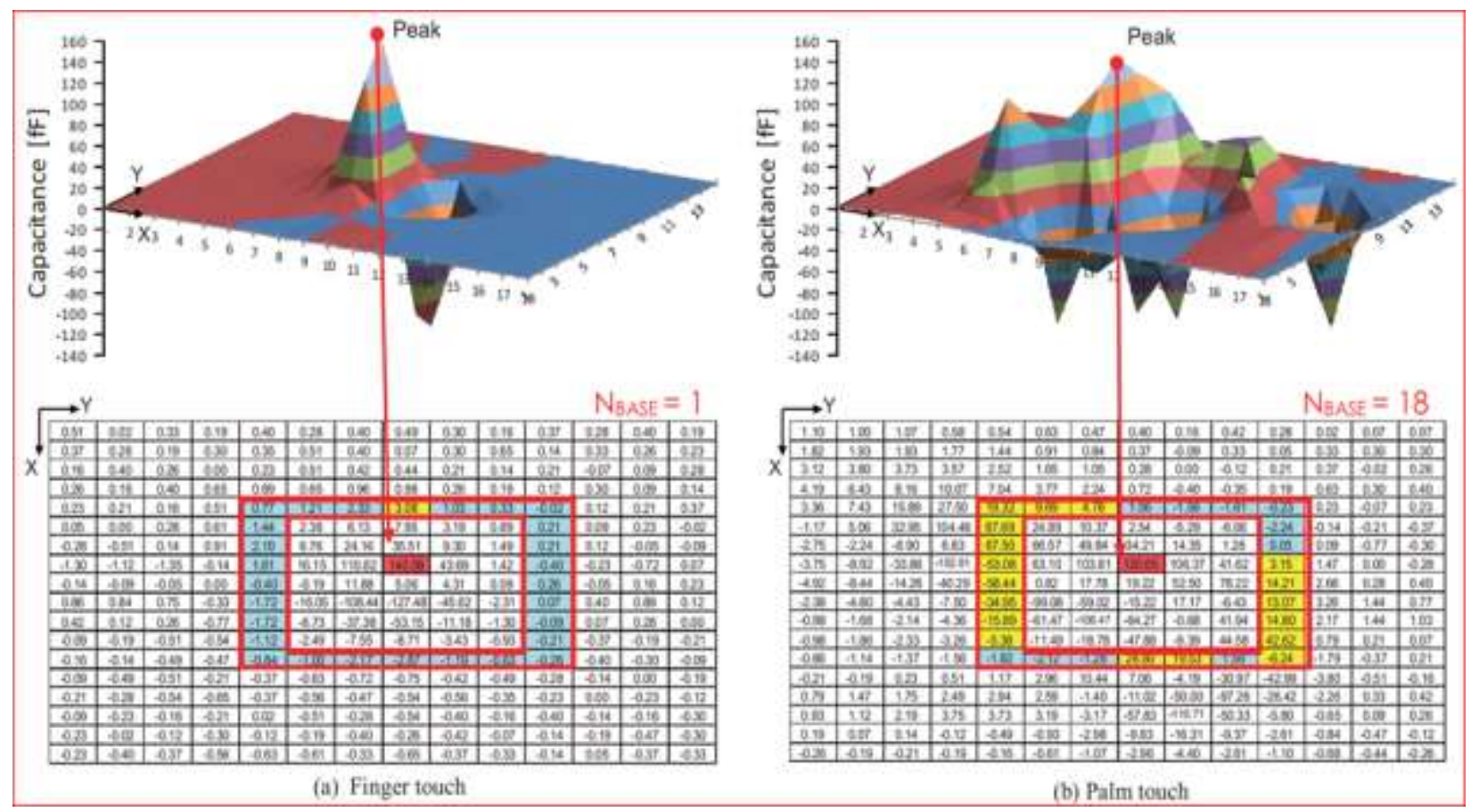

Figure 8 Touch sensor's capacitance profiles of filter method

\section{Conclusion}

In the paper, the whole functional modules of a touch screen system are first shown clearly. In addition, we defined the problem of palm rejection in touchscreen system and shown numerous solutions based on AFE, sensors, FFT, MCU, and hybrid solutions between them. The authors hope that the proposed research will be the good references for system on chip design engineer and research scientists on touch screen controller. 


\section{Compliance with ethical standards}

\section{Acknowledgments}

This work is supported by FPT University, Hanoi, Vietnam

Disclosure of conflict of interest

On behalf of all authors, corresponding author declares that there is no conflict of interest to publish this research.

\section{References}

[1] Nguyen DM, Kim S. "A novel construction for quantum stabilizer codes based on binary formalism". Int. J. of Modern Phys. B. 2020; 33(8).

[2] Hieu VD et. al. "Design and verification of novel classical error control codes using VERILOG Hardware Description Language". Int. J. of Advanced Trends in Computer Science and Engineering. 2020; 9(4).

[3] Geoff Walker, Palm rejection on resistive touchscreens.

[4] OK Kwon et.al. "Capacitive Touch Systems with Styli for Touch sensors: A Review." IEEE Sensors journal. 2018; 18(12).

[5] S Yoshida et.al. "An 87*49 mutual capacitance touch sensing IC enabling $0.5 \mathrm{~mm}$-diameter stylus signal detection at $240 \mathrm{~Hz}$-reporting rates with palm rejection". IEEE Asian Solid-State Circuits Conference. 2014. 